\section{Congenital Chloride Diarrhea in a Bartter Syndrome Misdiagnosed Brazilian Patient}

\section{Abstract}

The differential diagnosis in children with hypokalemic hypochloremic alkalosis include a group of an inherited tubulopathies, such as Bartter Syndrome (BS) and Gitelman Syndrome (GS). However, some of the clinically diagnosed patients present no pathogenic mutation in BS/GS known genes. Therefore, one can conclude that a similar clinical picture may be caused by PseudoBartter Syndrome (PBS) conditions. PBS include acquired renal problems (ex.: use of diuretics) as well as genetic or acquired extrarenal problems such as cystic fibrosis or cyclic vomiting, respectively. The accurate diagnosis of BS/GS needs a rational investigation. First step is to rule out PBS and confirm the primary renal tubular defect. However, it is not easy in some situations. In this sense, we reported a patient that was referred to our service with the diagnosis of BS, but presented no mutation in BS/GS known genes. The whole-exome sequencing detected a SCL26A3 likely pathogenic mutation leading to the final diagnosis of Congenital Chloride Diarrhea (CCD). Reviewing the records, the authors noticed that liquid stools were mistaken for urine. In addition, urinary and fecal samples were collected at an inappropriate time. The fecal sample taken during dehydration led to a low fecal chloride level and urine taken soon after sodium and potassium chloride infusion led to a high urinary chloride fractional excretion. Based on this data, BS was misdiagnosed. Our goal is to report the first Brazilian CCD patient initially misdiagnosed as BS and discuss the difficulties to achieve the correct diagnosis in clinical practice.

Keywords: Alkalosis; Child; Bartter syndrome; Gitelman syndrome; Congenital chloride diarrhea; Hypokalemia

Abbreviations: BS: Bartter Syndrome; GS: Gitelman Syndrome; PBS: PseudoBartter Syndrome; CCD: Congenital Chloride Diarrhea; CFTR: Cystic Fibrosis Transmembrane Conductance Regulator

\section{Maria Helena Vaisbich*, Juliana Caires de Oliveira Achili Ferreira, Ana Carola Hebbia Lobo Messa and Fernando Kok}

\author{
Department of Pediatric Nephrology, \\ Instituto da Criança, University of São Paulo, \\ São Paulo, Brasil
}

\section{*Corresponding author: \\ Maria Helena Vaisbich}

Department of Pediatric Nephrology, Instituto da Criança, University of São Paulo, São Paulo, Brasil.

”maria.vaisbich@hc.fm.usp.br

Tel: $+55-11-99940-3433$

Citation: Vaisbich MH, Ferreira JCDOA, Messa ACHL, Kok F (2019) Congenital Chloride Diarrhea in a Bartter Syndrome Misdiagnosed Brazilian Patient. J Rare Disord Diagn Ther. Vol.5 No.2:4

Received: August 16, 2019; Accepted: November 11, 2019; Published: November 18, 2019

\section{Introduction}

General pediatricians are frequently the first professionals to detect metabolic and electrolyte disturbances in childhood and they should be aware of the differential diagnosis. In cases of persistent hypochloremic hypokalemic metabolic alkalosis, acquired situations such as cyclic vomiting, chloride-intake deficiency, laxative abuse, diuretics use or nephrotoxic agents have to be investigated [1]. However, the late diagnosis could be harmful to patients with some rare genetic diseases [2]. In this context, the differential diagnosis of persistent hypochloremic hypokalemic metabolic alkalosis with high serum renin and aldosterone in childhood include a group of an autosomal recessive salt-loss tubulopathies, such as Bartter Syndrome (BS) and Gitelman Syndrome (GS) [3]. However, variants in known BS/GS genes have not been identified in some patients [4] suggesting other rare diseases may cause a PseudoBartter Syndrome (PBS). Table 1 summarizes the main differential diagnosis of this metabolic and electrolyte disturbance and provides some details of the etiologies. The clinical diagnosis of BS can be achieved with a rational investigation excluding PBS and confirming the primary renal tubular dysfunction. However, it is not easy in some situations. Congenital Chloride Diarrhea (CCD), a disease characterized by fecal chloride waste, can 


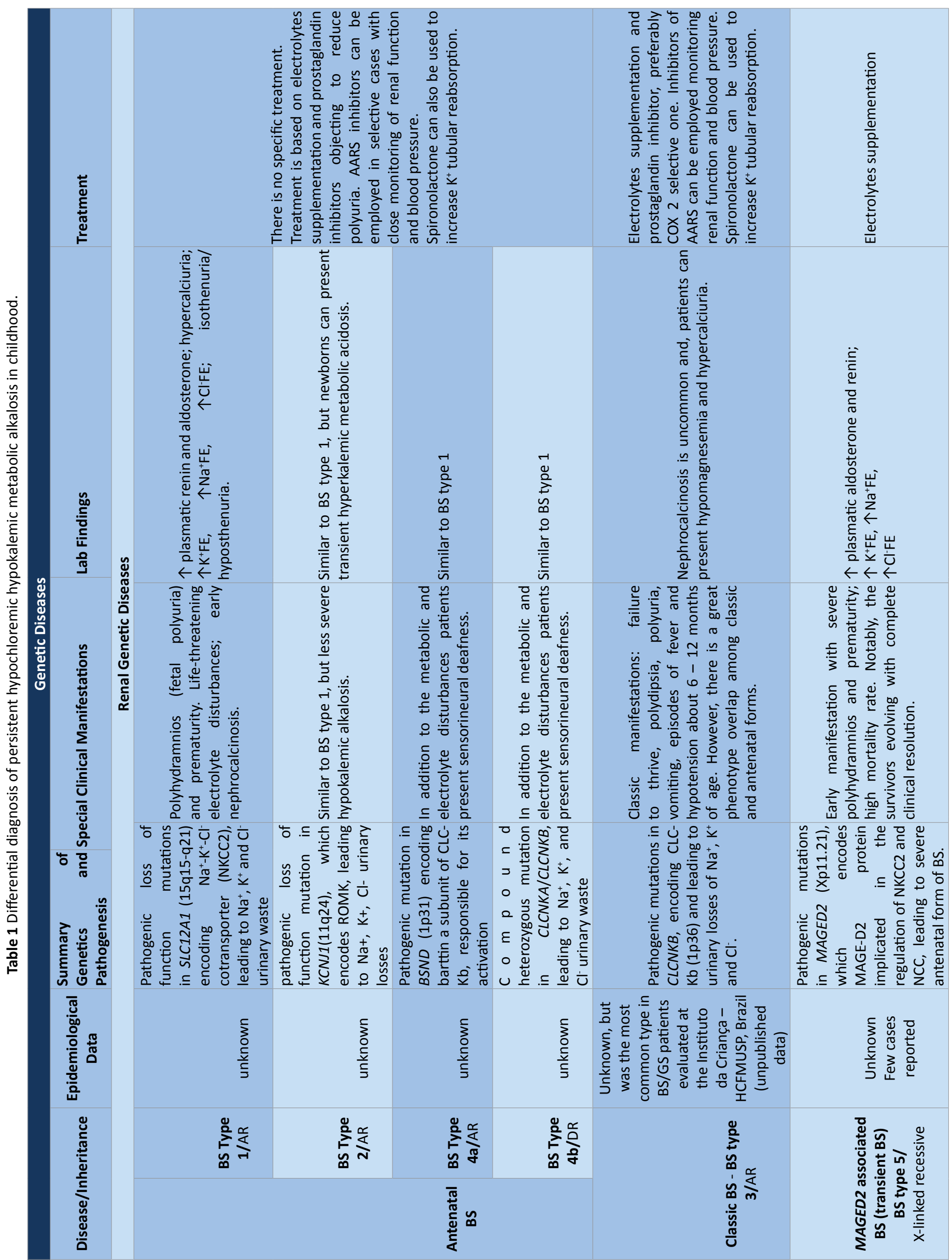




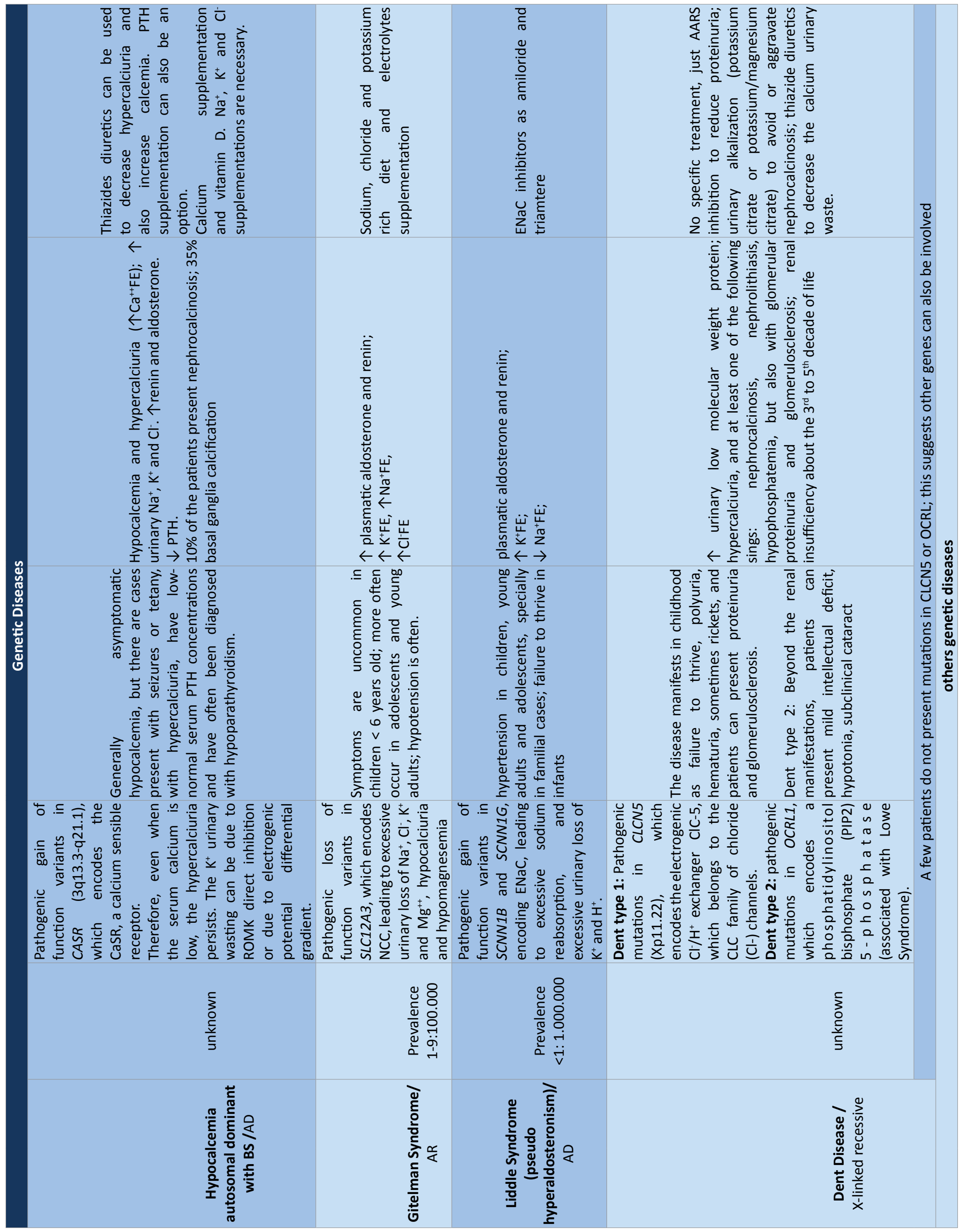




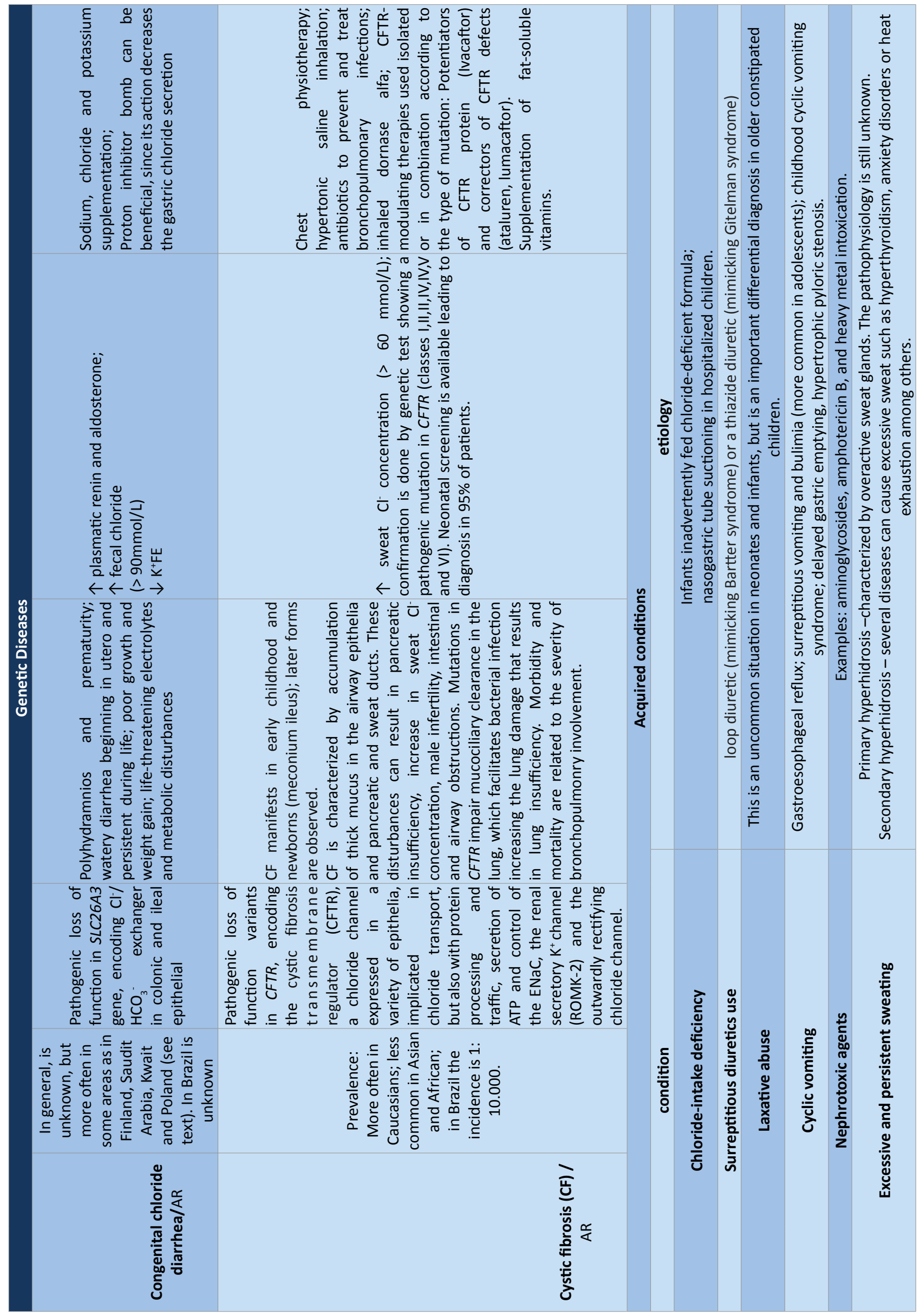


cause similar biochemical abnormalities and, in these patients, diarrhea can be confounded with polyuria, making this disease difficult to be suspected [5]. According to a recent publication of Ben-David et al., there are 13 case reports in literature about CCD patients initially misdiagnosed as BS, 10 from Middle East, 2 from Japan and 1 Serbian [6]. In this paper, the authors report the first Brazilian patient with CCD who was misdiagnosed as BS and the diagnosis was achieved just by Whole-Exome Sequencing (WES). Our goal is to discuss the difficulties to achieve the diagnosis in clinical practice.

\section{Case Report}

A female patient was born from healthy non-consanguineous parents at 36 weeks of gestation with an appropriate birth weight of $3420 \mathrm{~g}$. She had non-remarkable familial history. In the third trimester of gestation, polyhydramnios was observed. Hospital records reported polyuria and episodes of vomiting. At the fourth day of life she developed jaundice and the exams showed indirect bilirubin of $23 \mathrm{mg} / \mathrm{dl}$ and blood type $\mathrm{O} \mathrm{Rh}+$, while maternal blood type was A Rh+. ABO incompatibility was diagnosed and exsanguinous transfusion indicated. For this reason, other exams were collected and unexpectedly showed metabolic alkalosis $\left(\mathrm{pH}=7.6 \mathrm{pCO}_{2}=36.5 \mathrm{mmHg} \mathrm{HCO}_{3}{ }^{-}=36.3 \mathrm{mEq} / \mathrm{L} \mathrm{BE}=\right.$ +15.1), hyponatremia (125 mEq/L), hypokalemia (2.9 mEq/L) and hypochloremia (90 $\mathrm{mEq} / \mathrm{L}$ ). Unfortunately, simultaneous urinary electrolyte concentration was not checked. A surgical problem was ruled out. She was discharged on the 21st day of life, after jaundice resolution and serum sodium, potassium and chloride improvement with oral supplementation. The patient was followed up on an outpatient basis, being treated with oral potassium chloride supplementation ( $2-3 \mathrm{mEq} / \mathrm{kg} /$ day) with serum potassium range of 2.5 to $3.2 \mathrm{mEq} / \mathrm{L}$. During follow up, she was appropriately oral fed and the mother reported that the patient had pasty stools without blood or mucus about 2 times/ day and polyuria. No mental or other congenital defects were observed. She evolved with failure to thrive, but with adequate neuropsychomotor development. The investigation showed normal chloride levels in the sweat and normal functional coprology. Infectious diarrhea and food allergy or intolerance was ruled out. At 16 months of age, she was presented at emergency service with severe watery diarrhea, vomiting and dehydration, and the exams showed serum (s) $\mathrm{Na}^{+}=130 \mathrm{mEq} / \mathrm{L}$, $\mathrm{sK}^{+}=2.5 \mathrm{mEq} / \mathrm{L}, \mathrm{sCl}=91 \mathrm{mEq} / \mathrm{L}$, blood $\mathrm{pH}=7.58$ and $\mathrm{HCO}_{3}^{-}=$ $44 \mathrm{mEq} / \mathrm{l}$. At this time, an investigation about the pathway of electrolyte waste was finally proposed. Then, a sample of stool was collected, and the fecal electrolyte measurements were: $\mathrm{Na}^{+}=9.5 \mathrm{mEq} / \mathrm{L}, \mathrm{K}^{+}=8.95 \mathrm{mEq} / \mathrm{L}$ and $\mathrm{Cl}^{-}=40.3 \mathrm{mEq} / \mathrm{L}$. The patient received sodium and potassium chloride infusion. Immediately after volume recovery, a urine sample was taken for examination and showed: $\mathrm{Na}^{+}=77 \mathrm{mEq} / \mathrm{L}, \mathrm{K}^{+}=68 \mathrm{mEq} / \mathrm{L}$ and $\mathrm{Cl}^{-}=61 \mathrm{mEq} / \mathrm{L}$. The investigation detected elevated plasma renin $=15 \mathrm{ng} / \mathrm{ml}$ (normal: 0.51-2.64) and aldosterone $=95 \mathrm{ng} / \mathrm{dL}$ (normal: up to 39,2 ). All this information was obtained from the medical records, checking the time of each procedure, and can be susceptible to error. CCD was suspected since the patient was being treated for chronic hypochloremic metabolic alkalosis and was in the emergency room due to watery diarrhea. However, as CCD diagnosis criteria includes fecal chloride $>90 \mathrm{mEq} / \mathrm{l}$ and low urinary chloride [7] this hypothesis was ruled out and the patient was referred to our pediatric nephrology service with provisional diagnosis of BS. Supporting this possible diagnosis, the patient presented polyhydramnios, premature delivery, reported polyuria and characteristic lab findings. Unfortunately, the diagnosis of BS was accepted and new urinary electrolytes were not measured. She was treated with potassium chloride, indomethacin (later replaced by celecoxib) and omeprazole to avoid gastric symptoms. During follow up she presented rare episodes of mild diarrhea, metabolic and electrolyte control, adequate growth and weight gain. We performed genetic evaluation of our clinically diagnosed BS/GS patients, including the following genes: GLA, CLCNKA, CLCNKB, BSND, KCNJ1, SLC12A1, CTNS, AQP2, AVPR2, SLC12A3, CLDN19, CNNM2, CLDN16, TRPM6, SCNN1G, SCNN1B, ATP6V1B1, ATP6VOA4, SLC4A4. She had no mutation detected and WES was done revealing a homozygous mutation (c.1487 T>G; p.Leu496Arg) in the solute carrier family 26 (SLC26A3) on chromosome 7q31, encoding for a transmembrane $\mathrm{Cl} / \mathrm{HCO}_{3}$ exchanger mainly expressed in the apical brush border of ileum and colonic epithelium [8]. This variant was confirmed by Sanger sequencing. This mutation is likely pathogenic and was reported at least once related to CCD in a family from Hong Kong [8]. Celecoxib was withdrawn and the patient has evolved well under oral potassium chloride supplementation and omeprazole. She has no sign of renal dysfunction.

\section{Discussion}

The differential diagnosis of persistent hypochloremic hypokalemic metabolic alkalosis in childhood include some acquired conditions as well as genetic diseases that pediatricians must be aware of, in which the precise diagnosis is essential due to the life-threatening situation. The lack of knowledge of the differential diagnosis and an inappropriate investigation of this metabolic electrolyte disturbance can lead to misdiagnosis and, consequently, incorrect treatment and complications. This is exactly what happened to this patient that received indomethacin or celecoxib. This mistake could have led to renal dysfunction [9]. Table 1 shows possible diagnosis of this condition and details some factors that can guide the investigation. The diagnosis can vary according to the age and the clinical and familial history can rule out possibilities such as drug effects. Figure 1 suggests a way to investigate these patients. Especially in neonates and infants, information about prenatal and delivery conditions are important. For instance, gastric suction can cause metabolic alkalosis. Polyhydramnios associated with dilation of bowel loop in gestational ultrasonography can lead to CCD suspicion [10]. Metabolic alkalosis in neonates and young infants can be due to surgical problems such as hypertrophic pyloric stenosis and these issues must be ruled out at presentation [11]. The presence of early manifestations such as premature labor, polyhydramnios, and failure to thrive increases the probability of genetic causes. The basis of the investigation is to identify the pathway of electrolyte waste. This includes electrolyte serum and urinary measurements, but these have to be done after 


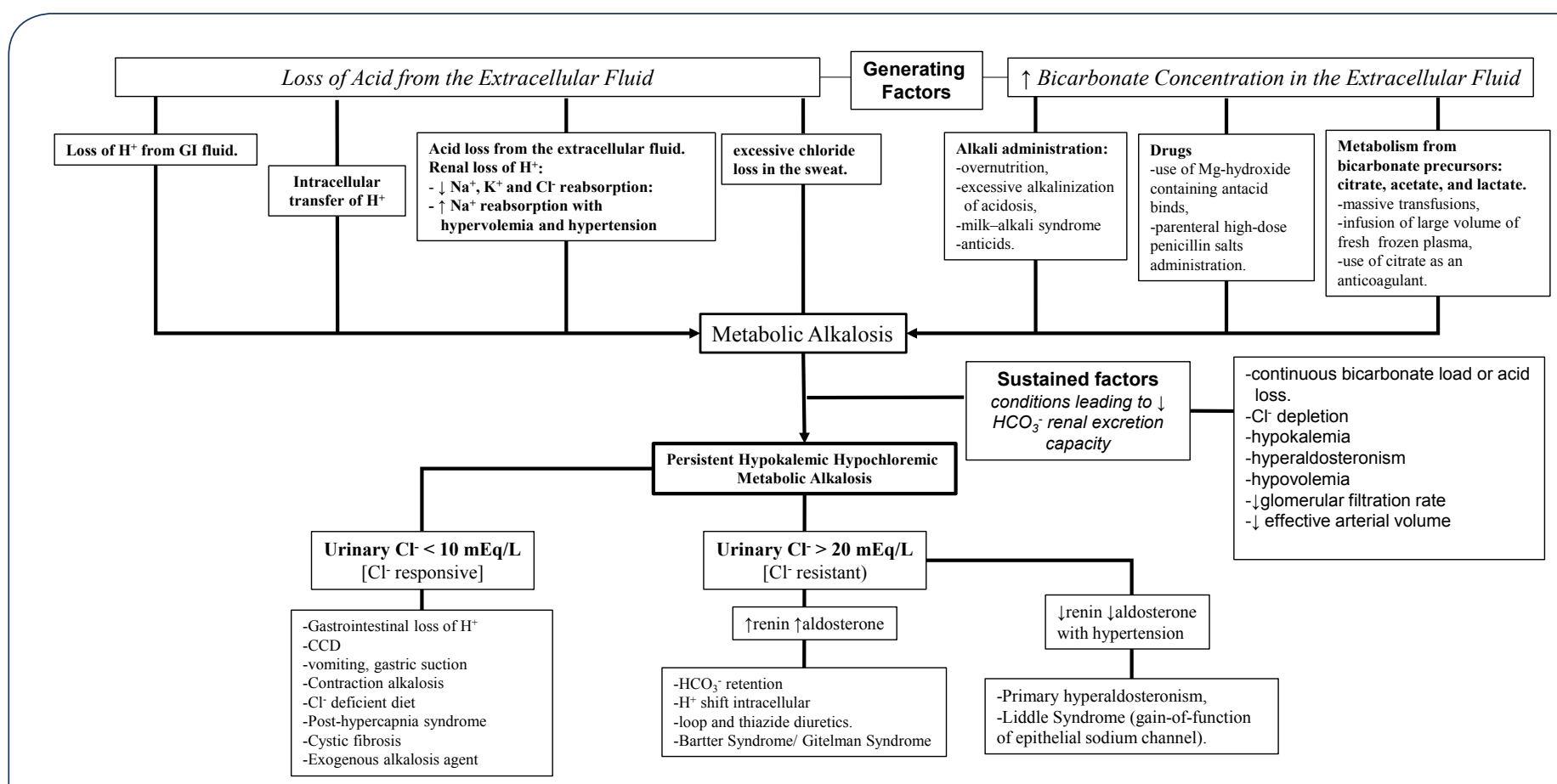

volume stabilization and without an intravenous overload of $\mathrm{NaCl}$ and $\mathrm{KCL}$, which can influence the results. In the presence of non-urinary waste, a PBS condition is suspected and chloride loss from other places such as skin or gastrointestinal tract has to be evaluated. CCD (OMIM 214700) is an autosomal recessive disease, that presents in the uterus with polyhydramnios (result of fetal diarrhea), which can cause slightly preterm birth (generally around 2 weeks before term) [12]. Therefore, CCD should be considered in cases of polyhydramnios and premature delivery. In cases that diarrhea is early recognized CCD is easily suspected, but the watery diarrhea often is misinterpreted as polyuria [12] especially in diaper babies. The disease is caused by mutations in the solute carrier family 26, member 3 gene (SLC26A3), on chromosome 7q31, encoding for a transmembrane $\mathrm{Cl} / \mathrm{HCO}_{3}^{-}$[13]. This protein coordinates the chloride absorption and bicarbonate secretion in the gastrointestinal tract. Loss-offunction mutations in SLC26A3 determine chloride accumulation in the intestinal lumen and $\mathrm{HCO}_{3}^{-}$is retained in the blood, resulting in watery $\mathrm{Cl}^{-}$rich diarrhea, hypochloremia, hypokalemia, and metabolic alkalosis [13]. Hypokalemia is a result of intestinal loss, but renal loss also occurs due to chloride depletion, hypovolemia and hyperactivation of the renin angiotensin aldosterone system. Secondarily, defective $\mathrm{Na}^{+} / \mathrm{H}^{+}$exchangers (NHE2 and/or NHE3) can lead to intestinal loss of $\mathrm{NaCl}$ and fluid, aggravating the diarrhea [13]. A close interaction between the cystic fibrosis transmembrane conductance regulator (CFTR) and SLC26A3 is supposed to be essential for epithelial $\mathrm{HCO}_{3}$ secretion. Interesting, at least two mutations in SLC26A3 prevent the activation of CFTR [13].

CCD is more common in some geographic areas. The estimated incidences in Finland and Poland are 1 in 10,000 and 1 in 200,000, respectively. Consanguineous marriages in Saudi Arabia and Kuwait cause high local incidences, such as 1 in 5,000 [8]. In Latin America, a novel homozygous likely pathogenic mutation was reported in a Mexican male in 2015 [14]. In Brazil, there is a lack of epidemiological data. Although the diagnosis of CCD seems to be simple, it cannot be predicted just on the basis of fecal chloride concentration, since it fluctuates depending on diet, hydration status, salt intake, and electrolyte balance. Volume and salt depletion may result in a low fecal $\mathrm{Cl}^{-}$of even $40 \mathrm{mEq} / \mathrm{l}$ [15]. This is the supposed reason for low fecal chloride in this patient, since the fecal sample was collected soon after the patient arrived in the emergency room while the patient was still dehydrated.

\section{Conclusion}

Low urinary chloride concentration is essential to confirm extrarenal chloride losses, gastrointestinal or skin. In this patient, the urinary sample was taken soon after sodium chloride and potassium infusion. This probably led to a transient increase in urinary chloride concentration, supporting the misdiagnosis of urinary chloride loss. Therefore, the collections should be done during volume stabilization and no electrolytes (sodium, potassium and chloride) intravenous administration which could influence the results. There are some reports identifying CCDrelated mutations in PBS patients. However, gene testing usually needs high cost and it is commonly unavailable. Therefore, it is important to keep in mind this uncommon diagnosis and proceed adequately to localize the place of electrolyte waste. Possibly, in near future some mutations could direct the treatment.

\section{References}

1 Matsunoshita N, Nozu K, Shono A, Nozu Y, Fu XJ, et al. (2016) 
Differential diagnosis of Bartter syndrome, Gitelman syndrome, and pseudo-Bartter/Gitelman syndrome based on clinical characteristics. Genet Med 18: 180-188.

2 Qiu L, Yang L, He Y, Yuan H, Zhou J (2018) Clinical characterization and diagnosis of cystic fibrosis through exome sequencing in Chinese infants with Bartter-syndrome-like hypokalemia alkalosis. Front Med 12: 550-558.

3 Seyberth HW, Schlingmann KP (2011) Bartter- and Gitelman-like syndromes: salt-losing tubulopathies with loop or DCT defects. Pediatr Nephrol 26: 1789-1802.

4 Matsunoshita N, Nozu K, Yoshikane M, Kawaguchi A, Fujita N, et al. (2018) Congenital chloride diarrhea needs to be distinguished from Bartter and Gitelman syndrome. J Hum Genet 63: 887-892.

5 Saneian H, Bahraminia E (2013) Congenital chloride diarrhea misdiagnosed as pseudo-bartter syndrome. J Res Med Sci 18: 822-824.

6 Ben-David Y, Halevy R, Sakran W, Zehavi Y, Spiegel R (2019) The utility of next generation sequencing in the correct diagnosis of congenital hypochloremic hypokalemic metabolic alkalosis. Eur J Med Genet 62: 103728.

7 Kamal NM, Khan HY, El-Shabrawi MHF, Laila M, Sherief LM (2019) A single center experience in a highly consanguineous population. Medicine 98: (e15928).

8 Hoglund P, Auranen M, Socha J, Popinska K, Nazer H, et al. (1998) Genetic Background of Congenital Chloride Diarrhea in High-
Incidence Populations: Finland, Poland, and Saudi Arabia and Kuwait. Am J Hum Genet 63: 760-768.

9 Winkelmayer WC, Waikar SS, Mogun H, Solomon DH (2008) Nonselective and cyclooxygenase-2-selective NSAIDs and acute kidney injury. Am J Med 121: 1092-1098.

10 Kawamura T, Nishiguchi T (2017) Congenital Chloride Diarrhea (CCD): A Case Report of CCD Suspected by Prenatal Ultrasonography and Magnetic Resonance Imaging (MRI). Am J Case Rep 18: 707-713.

11 Acker SN, Garcia AJ, Ross JT, Somme S (2015) Current trends in the diagnosis and treatment of pyloric stenosis. Pediatr Surg Int 31: 363-366.

12 Wedenoja S, Hoglund P, Holmberg C (2010) Review article: the clinical management of congenital chloride diarrhoea. Aliment Pharmacol Ther 31: 477-485.

13 Wedenoja S, Pekansaari E, Hoglund P, Makela S, Holmberg C, et al. (2011) Update on SLC26A3 mutations in congenital chloride diarrhea. Human Mutation 32: 715-722.

14 Reimold FR, Balasubramanian S, Doroquez DB, Shmukler BE, Zsengeller ZK, et al. (2015) Congenital chloride-losing diarrhea in a Mexican child with the novel homozygous SLC26A3 mutation G393W. Front Physiol 6: 179.

15 Amato F, Cardillo G, Liguori R, Scorza M, Comegna M, et al. (2017) Twelve novel mutations in the SLC26A3 gene in 17 sporadic cases of Congenital Chloride Diarrhea. J Pediatr Gastroenterol Nutr 65: 26-30. 